Geopolitics and geophilosophy: the place of Ibero-America in the world

\title{
Geopolitica y geofilosofía: el lugar de Iberoamérica en el mundo
}

\author{
Antonio Campillo \\ Universidad de Murcia \\ campillo@um.es \\ DOI: http://doi.org/10.15366/bp2019.22.024 \\ Bajo Palabra. II Época. № 22. Pgs: 443-458
}

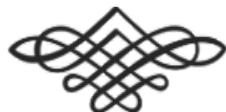


Recibido: 06/07/2019

Aprobado: 02/10/2019

Resumen

La filosofía debe asumir la paradoja que la constituye: tiene una clara vocación de universalidad, pero al mismo tiempo se inscribe siempre en un contexto espacial y temporal concreto. Con ocasión de la creación de la Red Iberoamericana de Filosofía (RIF), que pretende defender los estudios de Filosofía en el sistema educativo, vertebrar a la comunidad filosófica iberoamericana y promover a escala mundial el pensamiento en español y portugués, en este artículo se esboza una reflexión geopolítica y geopolítica sobre los muchos estratos históricos que han configurado el espacio cultural iberoamericano y sobre la necesidad de que cuente con instituciones filosóficas como la RIF, para contrapesar la hegemonía geopolítica y geofilosófica del espacio cultural anglo-americano y el creciente poder del espacio índico-pacífico.

Palabras clave: Iberoamérica, geopolitica, geofilosofía, español, portugués.

\section{Abstract}

Philosophy must assume the paradox that constitutes it: it has a clear vocation for universality, but at the same time it is always inscribed in a specific spatial and temporal context. On the occasion of the creation of the Red Iberoamericana de Filosofía (RIF), which aims to defend Philosophy studies in the educational system, to structure the Ibero-American philosophical community and promote thought in Spanish and Portuguese on a global scale, this article outlines a geopolitical and geopolitical reflection on the many historical strata that have shaped the Ibero-American cultural space and on the need to have philosophical institutions such as the RIF, to counterbalance the geopolitical and geophilosophical hegemony of the Anglo-American cultural space and the growing power of space Indico-Pacific.

Keywords: Ibero-America, geopolitics, geophilosophy, Spanish, Portuguese. 
La filosofía es Constitutivamente paradójica. Por un lado, tiene una clara vocación de universalidad, en la medida en que está abierta a todos los seres humanos. Por otro lado, se encuentra siempre históricamente situada en un lugar y un tiempo dados, lo que da origen a la diversidad de las filosofías y a la disputa entre ellas.

Hemos de reconocer el carácter insoluble de esta paradoja y afrontarla con la mayor lucidez posible. No podemos aspirar a la universalidad sin tener en cuenta que toda filosofía está ineludiblemente situada. Y, a la inversa, no podemos ser conscientes de nuestra propia situación si no la ponemos a prueba en el debate abierto con los otros.

Nos hemos congregado en este Simposio personas que procedemos de distintos países y tenemos distintas biografías, pero que al mismo tiempo compartimos la vocación por la filosofía y el marco cultural iberoamericano. Y nos hemos reunido porque queremos constituirnos como un actor colectivo en un mundo globalizado en el que, sin embargo, no impera el universalismo cosmopolita, sino más bien relaciones de dominación política, desigualdad económica y hegemonía cultural entre los pueblos.

En mi ponencia, me limitaré a enumerar brevemente los acontecimientos que considero cruciales para comprender la historia común de la que procedemos y los retos que hemos de afrontar en el futuro como Red Iberoamericana de Filosofía. Seguiré un procedimiento arqueológico, para poder mostrar que el actual territorio iberoamericano está formado por una serie de estratos culturales que se fueron sedimentando en sucesivas épocas históricas y que siguen condicionando el tiempo presente.

\footnotetext{
Una primera versión de este texto fue presentada el 12 de noviembre de 2018 en el Simposio de la Red Iberoamericana de Filosofía (RIF) "Cinco siglos de filosofía iberoamericana: problemas y retos actuales", coordinado por Ambrosio Velasco, en el marco del XIX Congreso Internacional de Filosofía "Mundo, Pensamiento, Acción”, organizado por la Asociación Filosófica de México (AFM) y celebrado en la Universidad Autónoma de Aguascalientes (México), del 12 al 16 de noviembre de 2018.
} 


\section{II}

LA HISTORIA DE LA HUMANIDAD ha sido una historia de constantes desplazamientos espacio/temporales: el homo sapiens es un homo viator, los humanos no tenemos raíces sino pies, somos animales constitutivamente migratorios, no hemos cesado de movernos de un lugar a otro, adaptándonos a los ecosistemas más diversos, enfrentándonos unos con otros, pero también mezclándonos y formando nuevas agrupaciones humanas. Son las migraciones geográficas las que han provocado las grandes mutaciones históricas.

Las primeras migraciones estuvieron protagonizadas por las más antiguas comunidades humanas, las sociedades tribales, que surgieron en África hace 200.000 años y comenzaron a migrar hace 65.000 años a la vecina Eurasia y desde allí a América y a Oceanía. Estos grupos nómadas realizaron la hazaña de poblar toda la Tierra y crear las instituciones más básicas de la convivencia humana. Hoy sabemos que todos los pueblos actuales somos parientes, todos tenemos antepasados negros, todos descendemos de los primeros migrantes africanos. El supuesto de que hay distintas razas humanas jerarquizadas entre sí carece de base científica. Nuestro común origen africano es el primer estrato arqueológico que compartimos todos los seres humanos.

Además, las sociedades tribales no sólo son las más antiguas sociedades humanas y las primeras en extenderse por toda la Tierra, sino que algunas han logrado perdurar hasta hoy, aunque se encuentran confinadas en lugares cada vez más aislados y amenazados ante el avance devastador del capitalismo globalizado. Actualmente hay unos 370 millones de personas indígenas y 40 millones de ellas siguen siendo nómadas.

En resumen, las sociedades tribales han protagonizado más del $95 \%$ de la historia humana. Sin embargo, la mayor parte de los filósofos y teóricos sociales del Occidente euro-atlántico las han menospreciado como "prehistóricas», "primitivas», «salvajes», «bárbaras», etc. Según Hegel, la Historia Universal de la Humanidad sólo comienza con la aparición del Estado, es decir, con los grandes imperios agrarios.

\section{III}

El SEGUNDO TIPO DE MIGRACIÓN y, por tanto, nuestro segundo estrato arqueológico lo protagonizan precisamente las sociedades con Estado. Aparecen en Mesopotamia hace 5.300 años y más tarde en Egipto, India, China, América central y andina, África subsahariana y algunas islas de Oceanía. Surgen como un archipiélago de 
Estados-ciudad, hasta que uno de ellos conquista a sus vecinos y se transforma en un Estado-imperio. Algunos imperios fueron muy extensos, como China y Roma en Eurasia, Malí en África, Maya en América o Tu’i Tonga en Oceanía, pero en general se ignoraban entre sí y todos colapsaron por crisis internas o por el auge de otros imperios.

Sin embargo, en el continente euroasiático, por ser el más extenso ${ }^{2}$ y por sus características ecológicas -al estar situado en la zona templada del hemisferio norte y orientado longitudinalmente entre el este y el oeste-, se crearon redes de comunicación horizontal entre el Oriente pacífico y el Occidente atlántico -por tierra la ruta de las caravanas y por mar las rutas del Índico y el Mediterráneo-, que también conectaron a Eurasia con África del norte y del este, permitiendo así el movimiento y la mezcla de personas, animales, plantas, microbios, enfermedades, técnicas, idiomas, ideas, etc.

En cambio, el continente americano, aunque es el segundo más extenso ${ }^{3}$, está orientado latitudinalmente entre los dos polos terrestres, por lo que la comunicación vertical entre el norte y el sur ha sido siempre más difícil. Algunos geógrafos e historiadores actuales ${ }^{4}$ consideran que estas diferencias ecológicas son las que permitieron a los pueblos europeos acumular los recursos biológicos, tecnológicos y políticos necesarios para conquistar las tierras de los pueblos americanos y no a la inversa. En cualquier caso, hasta 1492, el Atlántico y el Pacífico mantuvieron a los pueblos de América casi completamente separados de los de Eurasia, África y Oceanía.

\section{IV}

Ahora bien, en el CONTINENTE euroasiático se produjo una gran división entre el Oriente y el Occidente, entre Asia y Europa. La civilización occidental o europea se inicia hace unos 2.500 años mediante su confrontación geopolítica con el Oriente asiático y el Sur africano. Es entonces cuando se forja el mito del «milagro griego", según el cual el logos y la pólis nacieron en Grecia como Atenea de la cabeza de Zeus, sin precedente histórico alguno y sin paralelo en otras tradiciones culturales, de modo que sólo en ese lugar y ese tiempo se produjo el tránsito de

\footnotetext{
${ }^{2}$ Eurasia tiene 55 millones de $\mathrm{km}^{2}$, el 10,6\% del planeta y el 36,2\% de la zona terrestre, unos 5.000 millones de habitantes en 91 países, el $72,5 \%$ de la población mundial y una densidad de 88,5 hab. $/ \mathrm{km}^{2}$.

3 América tiene 43,3 millones de $\mathrm{km}^{2}$, el 8,4\% del planeta y el 30,2\% de la zona terrestre, más de 1.000 millones de habitantes en 35 países, el $12 \%$ de la población mundial y una densidad de $24,03 \mathrm{hab} . / \mathrm{km}^{2}$.

${ }^{4}$ Crosby, A. W., Imperialismo ecológico. La expansión biológica de Europa, 900-1900, Barcelona, Crítica, 1988, orig. 1986; Diamond, J., Armas, gérmenes y acero. Breve historia de la humanidad en los últimos trece mil años, Madrid, Debate, 2004, orig. 1997.
} 
la narración fabulosa a la argumentación racional, pero también de la tiranía a la libertad, en resumen, de la barbarie a la civilización ${ }^{5}$. Este relato fundacional es el gran mito eurocéntrico del pensamiento occidental. Derrida lo definió como la «mitología blanca» ${ }^{6}$. La filosofía griega nace como una "geofilosofía» vinculada a una "geopolítica»: desde que las ciudades helenas derrotaron al imperio persa, su cultura se expandió por todo el Mediterráneo y, con ella, el mito de la superioridad del Occidente libre sobre el Oriente despótico. La idea de Europa como cuna de la civilización tiene aquí su origen. ${ }^{7}$

El imperio romano y la iglesia católica heredaron la «mitología blanca» creada por los griegos y la desarrollaron mediante la teoría de la translatio imperii et studii. Según esta teoría, en la historia humana se ha dado un desplazamiento geográfico de los imperios y de la sabiduría que ha seguido el curso del sol, de Oriente a Occidente y del Índico al Atlántico. Esta teoría recorre la historia de Europa, de san Agustín a Hegel. ${ }^{8}$ En España, la idea de la translatio imperii et studii la retoman en el siglo XV varios humanistas, entre ellos Antonio de Nebrija (1441-1522), autor de la primera Gramática sobre la lengua castellana, publicada en Salamanca en 1492, el mismo año en que los Reyes Católicos unifican los reinos hispanos y Colón llega a América. Nebrija dedica el libro a Isabel la Católica, reina de Castilla, y en el Prólogo le dice:

Cuando bien comigo pienso, mui esclarecida reina, i pongo delante los ojos el antigüedad de todas las cosas que para nuestra recordación i memoria quedaron escriptas, una cosa hallo i saco por conclusión mui cierta: que siempre la lengua fue compañera del imperio i de tal manera lo siguió que junta mente començaron, crecieron i florecieron i, después, junta fue la caída de entrambos.

(...) Después que vuestra Alteza metiesse debaxo de su iugo muchos pueblos bárbaros y naciones de peregrinas lenguas, y con el vencimiento aquellos ternían necessidad de recebir las leies quel vencedor pone al vencido, y con ellas nuestra lengua, entonces, por esta mi arte, podrían venir en el conocimiento della, como agora nos otros deprendemos el arte de la gramática latina para deprender el latin. ${ }^{9}$

\footnotetext{
5 Burnet, J., La aurora de la filosofía griega, México, Argos, 1944, orig. 1915.

6 Derrida, J., "La mitología blanca. La metáfora en el texto filosófico", en Márgenes de la filosofía, Madrid, Cátedra, 1989, pp. 247-311. Véase también Young, R. J. C., White Mitologies. Writing History and the West, Londres y Nueva York, Routledge, 1990, 2a ed. 2004.

7 Cacciari, M., Geofilosofía de Europa, Cuenca, Alderabán, 2001, y Europa o la Filosofía, Madrid, Antonio Machado, 2007; Duque, F., Los buenos europeos. Hacia una filosofía de la Europa contemporánea, Oviedo, Nobel, 2003; Nájera, E., y Pérez Herranz, F. M. (eds.), La filosofia y la identidad europea, Valencia, Pre-textos, 2011.

${ }^{8}$ Padua, M. de, Sobre el poder del imperio y del papa. El defensor menor. La transferencia del imperio, Madrid, Biblioteca Nueva, 2005; Goez, W., Translatio imperii, Tübingen, Mohr, 1958; León Florido, F., "Translatio studiorum: traslado de los libros y diálogo de las civilizaciones en la Edad Media”, en Revista general de información $y$ documentación, vol. 15, no 2, 2005, 51-77.

9 Nebrija, A. de, Gramática sobre la lengua castellana (1492), ed. de C. Lozano, y Paginae nebrissenses, ed. de F. González Vega, Madrid y Barcelona, RAE y Galaxia Gutenberg, 2011, p. 3.
} 
Con la llegada de Colón a América se inicia un nuevo movimiento migratorio y, con él, un nuevo estrato geológico de lo que hoy llamamos Iberoamérica. Se produce entonces el encuentro o, más bien, el «encontronazo»-como lo llamó Rafael Sánchez Ferlosio ${ }^{10}$ - de los pueblos europeos con los pueblos del continente americano.

La translatio imperii et studii siguió desplazándose hacia Occidente, pero ahora cruzando el Atlántico y legitimando los imperios ultramarinos, como hizo Hernán Cortés frente al imperio mexica y el Inca Garcilaso de la Vega frente al imperio inca.

A partir de entonces se crea la primera sociedad mundial de la historia y con ella una nueva forma de dominación global que conecta a todos los pueblos de la Tierra y da lugar a fenómenos como el comercio de esclavos negros entre Europa, África y América. Al mismo tiempo, se inicia la llamada Modernidad, con la que Europa pretende promover el progreso material, intelectual y moral de toda la humanidad.

En efecto, para legitimar la hegemonía mundial de la Europa atlántica y la superioridad de la época moderna sobre todas las épocas precedentes, las élites europeas elaboran una filosofía de la historia basada en la idea de progreso ${ }^{11}$. Fue Hegel quien formuló de manera más sistemática esta concepción evolutiva y eurocéntrica de la historia humana y del propio pensamiento filosófico. Por eso, algunos teóricos de los estudios "postcoloniales» han tomado a Hegel como la gran referencia polémica. ${ }^{12}$

En el nuevo espacio/tiempo de la moderna sociedad capitalista, se dan tres grandes desplazamientos geopolíticos y geofilosóficos:

1. El desplazamiento de la hegemonía mundial, que en los siglos XVII y XVIII pasa de los imperios coloniales de la Europa católica del sur (España y Portugal) a los de la Europa protestante del norte (Holanda, Francia y, sobre todo, Inglaterra). Este desplazamiento de la hegemonía afectó también a las colonias americanas y acabó condicionando toda la época moderna. Todavía hoy, la alianza militar más poderosa del mundo es la OTAN y está liderada por la ex colonia estadounidense. Además, este desplazamiento del sur al norte no sólo afectó al poder político sino también al poder cultural: los historiadores angloamericanos, además de difundir la «leyenda negra» sobre el imperio español, sitúan el inicio de la Modernidad en la Reforma protestante y no en el Renacimiento

\footnotetext{
${ }_{10}$ Sánchez Ferlosio, R., Esas Yndias equivocadas y malditas. Comentarios a la historia, Barcelona, Destino, 1994. Incluido en Ensayos y artículos, 2 vols., Barcelona, Destino, 1992, vol. II, pp. 517-803.

${ }^{11}$ Campillo, A., Adiós al progreso. Una meditación sobre la historia, Barcelona, Anagrama, 1985, 2a ed. 1995, y Variaciones de la vida humana. Una teoría de la historia, Madrid, Akal, 2001.

${ }^{12}$ Chakrabarty, D., Provincializing Europe: Postcolonial Thought and Historical Difference, Princeton / Oxford, Princeton U. P., 2000.
} 
católico; de este modo, la filosofía moderna comienza con el racionalismo francés, el empirismo inglés y el idealismo alemán, y no con los debates hispanos sobre la legitimidad de la conquista, ni con Maquiavelo, Galileo o Suárez.

2. El segundo gran desplazamiento de la época moderna se produce entre 1740 y 1830, con los movimientos de descolonización e independencia de las colonias americanas (Estados Unidos, Haití y los demás países del Caribe y América Latina). La gran paradoja de este proceso es que, por un lado, los pueblos americanos se emancipan de los imperios coloniales europeos; pero, por otro lado, las élites que protagonizan la independencia acaban aplicando en sus propios países las ideas y políticas europeas, sobre todo frente a la resistencia de las comunidades indígenas y campesinas. ${ }^{13}$

3. El tercer desplazamiento se produce en el siglo XIX. Aparecen tres nuevos movimientos sociales emancipatorios que denuncian las insuficiencias de la primera Declaración de Independencia (Estados Unidos, 1776) y la primera Declaración de los Derechos del Hombre y del Ciudadano (Francia, 1789), en la medida en que dejan fuera de los derechos de ciudadanía a la mayor parte de la población: a) el movimiento antiesclavista y antisegregacionista de las comunidades negras e indias; b) el movimiento obrero y socialista de los trabajadores asalariados; c) el movimiento sufragista y feminista de las mujeres (europeas y americanas, burguesas y trabajadoras).

\section{VI}

EN EL SIGLO XX CONTINÚAN los tres grandes desplazamientos de la época moderna (el traslado de la hegemonía mundial del sur al norte euro-atlántico, los procesos de descolonización de las colonias europeas en África, Asia y Oceanía, y los movimientos emancipatorios de las mujeres, los trabajadores y los pueblos subalternos), pero se producen también otros desplazamientos completamente nuevos:

1. La Guerra Civil Europea (1914-1945), que incluye guerras, revoluciones, deportaciones, genocidios, etc., con un saldo de 90 millones de muertos. En ese

\footnotetext{
13 Un ejemplo de esta paradoja es Domingo Faustino Sarmiento (1811-1888), uno de los políticos argentinos más importantes del siglo XIX y uno de los más fervientes defensores de la colonización de América por los europeos. Entre su ingente obra literaria y ensayística, destaca Facundo o Civilización y barbarie en las pampas argentinas (1845). Véase el artículo de Scavino, D., "Sarmiento y la translatio imperii", en Estudios de Teoría Literaria, ańo 5, no 10, septiembre 2016, pp. 167-177.
} 
ciclo se inscriben la Guerra Civil Española (1936-1939) y las largas dictaduras de Franco en España (1939-1975) y Salazar en Portugal (1926-1974), que provocan un importante exilio de intelectuales españoles y portugueses a Europa y América, contribuyendo así a crear una comunidad cultural iberoamericana de pensadores «desterrados» o "transterrados». Un exilio paralelo se produjo desde Europa central hasta América del Norte, sobre todo por parte de muchos judeo-alemanes. Todos estos exiliados europeos emprenden la gran autocrítica de la Modernidad occidental. Se produce así la crisis de la filosofía moderna que había prevalecido en Europa desde la conquista de América.

2. Después de 1945, Europa pierde la hegemonía mundial y pasan a disputársela dos nuevas superpotencias mundiales (Estados Unidos y la Unión Soviética), con sus bloques económicos y militares. Es el inicio de la Guerra Fría (1945-1991). El mundo se divide entonces en tres grandes áreas geopolíticas: el Primer Mundo (el Occidente capitalista), el Segundo Mundo (el Oriente comunista) y el Tercer Mundo (el Sur global, que pasa a ser el territorio disputado por los dos bloques del Norte).

3. Se inicia una nueva ola de descolonización en el Tercer Mundo, sobre todo en África y Asia, promovida por Estados Unidos y la Unión Soviética, lo que desencadena una serie de guerras civiles (Corea, Vietnam, Palestina, Angola, Sáhara, Afganistán...), y, en el caso de América Latina, golpes y dictaduras militares, guerrillas insurgentes, grupos paramilitares, cárteles de la droga, el bloqueo a Cuba, etc.

4. Paralelamente, el proceso de modernización de las ex colonias europeas del Tercer Mundo adopta la forma de una expansión del capitalismo global bajo el discurso del «desarrollo». Se divide a los países en una nueva jerarquía: «desarrollados», «en vías de desarrollo» y «subdesarrollados». Frente a esta jerarquía, surgen movimientos teóricos y prácticos que denuncian las relaciones de "dependencia» estructural entre el «desarrollo» del Norte enriquecido y el «subdesarrollo» del Sur empobrecido. Esta «dependencia» es cuestionada como una forma de "neocolonialismo", en la que participan las élites autoritarias y corruptas de los países del Sur, y cuyo resultado son los numerosos estragos sociales y ambientales sufridos en las últimas décadas. ${ }^{14}$

\footnotetext{
${ }^{14}$ Escobar, A., La invención del Tercer Mundo. Construcción y deconstrucción del desarrollo, prólogo de Orlando Fals Borda, Bogotá, Norma, 1996. Orig. inglés: Encountering Development. The making and unmaking of the Third World, Princeton, 1995. Según Escobar, "el modelo del desarrollo desde sus inicios contenía una propuesta
} 
5. Entre 1945 y 1991, hay también algunos fenómenos positivos: la creación de la ONU, la Declaración Universal de los Derechos Humanos, el proceso de construcción y ampliación de la Unión Europea -en la que España y Portugal, tras su transición democrática, ingresan en 1986-, la formación del Estado de bienestar, la segunda ola del movimiento feminista y, por último, a partir de 1961, el Movimiento de Países No Alineados en el Sur global, promovido por India, Egipto e Indonesia.

\section{VII}

La ÉPOCA ACTUAL SE INICIA TRAS I968, con el triunfo del neoliberalismo (Pinochet en 1973, Thatcher en 1979 y Reagan en 1981), el final de la Guerra Fría (1991) y el choque del capitalismo con los límites biofísicos del planeta, anunciado ya en 1972 por el informe Los límites del crecimiento. Recordaré algunos fenómenos relevantes:

1. Estados Unidos se convierte en la potencia hegemónica mundial. Pero, en contra de los ideólogos del "final de la historia»" ${ }^{15}$, esa hegemonía encubre su paulatino declive ${ }^{16}$. La crisis iniciada en 2007-2008 ha mostrado que se está produciendo un desplazamiento del centro geopolítico del mundo desde el Occidente euro-atlántico al Oriente asiático-pacífico, en donde se concentra ya la mitad de la población mundial. Es como si la translatio imperii et studii hubiera dado la vuelta a la Tierra y regresado de nuevo a Oriente. Así lo dicen expresamente los actuales estrategas chinos: el primer milenio fue de China, el segundo lo fue de Europa y el tercero lo será nuevamente de China. En realidad, ahora nos encontramos en un interregno en el que prevalece un nuevo desorden westfaliano a escala mundial, una multipolaridad global en la que ninguna potencia puede imponer su dominación imperial a las demás. Este cambio geopolítico está teniendo ya algunas consecuencias geofilosóficas: no es ninguna casualidad que las sedes de los Congresos Mundiales de Filosofía se hayan desplazado también en los últimos años (la translatio studii) desde las grandes capitales del Occidente euro-atlántico hacia las capitales de las

históricamente inusitada desde un punto de vista antropológico: la transformación total de las culturas y formaciones sociales de tres continentes de acuerdo con los dictados del llamado Primer Mundo". El libro narra cómo este "sueño" se transformó en "pesadilla".

15 Fukuyama, F., El fin de la historia y el último hombre, Barcelona, Planeta, 1992.

${ }_{16}$ Mann, M., El imperio incoherente. Estados Unidos y el nuevo orden internacional, Barcelona, Planeta, 2004; Todd, E., Después del imperio. Ensayo sobre la descomposición del sistema norteamericano, Madrid, Akal, 2012; Patiño Villa, C. A., Imperios contra Estados. La destrucción del orden internacional contemporáneo, Bogotá, Debate, 2017. 
nuevas potencias emergentes: Estambul (2003), Seúl (2008), Atenas (2013), Beijing (2018) y Melbourne (2023).

2. Este desplazamiento geopolítico y geofilosófico es compatible con la gran expansión mundial del capitalismo en su fase neoliberal, que está vinculada a la revolución de las TICs y la financiarización de la economía, pero también al incremento de las desigualdades Norte-Sur, el expolio de los recursos naturales, la producción masiva de desechos, el cambio climático antropogénico, las nuevas migraciones globales y las nuevas políticas xenófobas en los países receptores. Todas las grandes potencias -sean de Occidente como Estados Unidos o de Oriente como China-, a pesar de sus conflictos geopolíticos, aceptan la hegemonía global del capitalismo, y esto ha dado origen a la gran paradoja de una "globalización amurallada»: apertura total de fronteras para el capital y cierre total para los migrantes y refugiados sin recursos. ${ }^{17}$

3. La hegemonía del neoliberalismo está transformando también las formas de creación y transmisión del conocimiento. Es lo que se conoce como «capitalismo académico» ${ }^{18}$. Mencionaré sólo tres fenómenos: a) la reducción de la financiación pública de la educación, la ciencia y la cultura, y su creciente privatización o bien su gestión con criterios empresariales; b) la identificación del conocimiento con la triada $\mathrm{I}+\mathrm{D}+\mathrm{i}$, es decir, con las innovaciones tecnocientíficas susceptibles de ser patentadas y mercantilizadas, mientras que las artes, las humanidades, las ciencias sociales y la filosofía -con algunas excepciones como la industria del entretenimiento y las tareas ideológicas de los académicos neoliberales- son consideradas como económicamente no rentables y socialmente perturbadoras, así que las políticas educativas neoliberales van orientadas a su progresiva reducción o marginalización; c) por último, la imposición del inglés como lingua franca universal de la educación, la ciencia y la cultura. ${ }^{19}$

17 Michael Hart y Antonio Negri se refieren a este imperio global del capitalismo, distinto de los imperios territoriales y coloniales del pasado, en Imperio, Barcelona, Paidós, 2005. Me ocupo de la "globalización amurallada" en mis dos últimos libros: Tierra de nadie. Cómo pensar (en) la sociedad global, Barcelona, Herder, 2015, y Mundo, nosotros, yo. Ensayos cosmopoliéticos, Barcelona, Herder, 2018.

18 Sousa Santos, B. de, La universidad en el siglo XXI. Para una reforma democrática y emancipadora de la universidad, Buenos Aires, Miño y Dávila, 2005; Slaughter, S., y Leslie, L. L., Academic Capitalism: Politics, Policies, and the Entrepreneurial University, Baltimore, Johns Hopkins U P, 1997; Slaughter, S., y Rhoades, G., Academic Capitalism and the New Economy; Markets, State, and Higher Education, Baltimore, Johns Hopkins U P, 2009; Campillo, A., "La Universidad en la sociedad global", en Isegoría. Revista de Filosofía Moral y Política, 52, enero-junio 2015, pp. 15-42.

19 El número de hablantes, en millones, de las llamadas «lenguas mundiales»: inglés (1.500), español (560) y francés (285), las tres de origen europeo. Los hablantes de las lenguas «suprarregionales»: chino mandarín (1.345), 


\section{VIII}

EN RESUMEN, el ESPACiO geOpolítico y geofilosófico al que hoy llamamos Iberoamérica es el resultado de una serie de desplazamientos espacio/temporales que se han ido acumulando en el curso de la historia y que constituyen nuestro ineludible horizonte existencial, la situación a partir de la cual hemos de pensar y actuar.

España y Portugal, que hace cinco siglos crearon los primeros grandes imperios coloniales ultramarinos en el continente americano, hoy son dos Estados de tamaño mediano/pequeño situados en el Norte rico, concretamente en la Unión Europea, pero en ella ocupan una posición subalterna. Latinoamérica y el Caribe, que desde comienzos del siglo XIX se emanciparon de los imperios español y portugués, hoy son un heterogéneo grupo de Estados que han pasado a depender de las grandes potencias del Norte anglófono y del Oriente asiático, y no han sido capaces de federarse como región geopolítica para hacer valer sus intereses en el nuevo desorden mundial.

En este contexto, los países de América Latina, el Caribe y la Península Ibérica necesitamos aliarnos no sólo para preservar nuestro pasado común, sino también para actuar de forma coordinada en el gran escenario geopolítico de la sociedad global.

En cuanto al futuro de la filosofía iberoamericana, en mi opinión nos enfrentamos a tres grandes retos:

1. Construir vínculos institucionales, intelectuales y personales entre profesores, estudiantes e investigadores de ambos lados del Atlántico, para reivindicar nuestras lenguas, nuestro diversidad cultural y nuestra creatividad filosófica, frente a la hegemonía política, económica, lingüística y cultural de los países anglófonos.

2. Reivindicar la importancia del pensamiento filosófico en el sistema educativo, en las universidades, en la investigación, en la cultura y en los grandes debates de la vida pública, frente a las políticas neoliberales que pretenden imponer el «pensamiento único» o "pensamiento cero» mediante la reducción del conocimiento a las llamadas disciplinas STEM: ciencias, tecnologías, lengua inglesa y matemáticas.

3. Renovar profundamente la tradición filosófica para afrontar las grandes transformaciones del siglo XXI. Los movimientos intelectuales más innovadores, entre ellos las filosofías europeas que han llevado a cabo la autocrítica de la Modernidad occidental, los estudios «postcoloniales» o «decoloniales» de América Latina, África

hindustaní (490), árabe (450), portugués (220), ruso (300), alemán (180), malayo e indonesio (176/250), lenguas iraníes (150/200), suajili (100), tamil (77), italiano (70) y holandés (46). 
y Asia, las organizaciones «sin fronteras» defensoras de los derechos humanos, los estudios feministas y de género, el pensamiento ecologista y sus propuestas de transición ecosocial hacia una nueva civilización planetaria, etc., no sólo están cuestionando el paradigma evolutivo y eurocéntrico en cuyo marco ha sido contada en los últimos cinco siglos la historia de la humanidad, sino que también están tratando de pensar con otras categorías las grandes mutaciones de la sociedad global, para abrir nuevos horizontes vitales a los más de 7.600 millones que habitamos ya sobre la Tierra. 


\section{Referencias Bibliográficas}

Burnet, J., La aurora de la filosofía griega, México, Argos, 1944, orig. 1915.

Cacciari, M., Geofilosofía de Europa, Cuenca, Alderabán, 2001.

Cacciari, M., Europa o la Filosofía, Madrid, Antonio Machado, 2007.

Campillo, A., Adiós al progreso. Una meditación sobre la historia, Barcelona, Anagrama, 1985, 2a ed. 1995.

Campillo, A., Variaciones de la vida humana. Una teoría de la historia, Madrid, Akal, 2001.

Campillo, A., "La Universidad en la sociedad global", en Isegoría. Revista de Filosofia Moral y Politica, 52, enero-junio 2015, pp. 15-42.

Campillo, A., Tierra de nadie. Cómo pensar (en) la sociedad global, Barcelona, Herder, 2015.

Campillo, A., Mundo, nosotros, yo. Ensayos cosmopoliéticos, Barcelona, Herder, 2018.

Chakrabarty, D., Provincializing Europe: Postcolonial Thought and Historical Difference, Princeton / Oxford, Princeton U. P., 2000.

Crosby, A. W., Imperialismo ecológico. La expansión biológica de Europa, 9001900, Barcelona, Crítica, 1988, orig. 1986.

Derrida, J., "La mitología blanca. La metáfora en el texto filosófico", en Márgenes de la filosofía, Madrid, Cátedra, 1989, pp. 247-311.

Diamond, J., Armas, gérmenes y acero. Breve historia de la humanidad en los últimos trece mil años, Madrid, Debate, 2004, orig. 1997.

Duque, F., Los buenos europeos. Hacia una filosofia de la Europa contemporánea, Oviedo, Nobel, 2003.

Escobar, A., La invención del Tercer Mundo. Construcción y deconstrucción del desarrollo, prólogo de Orlando Fals Borda, Bogotá, Norma, 1996. Orig. inglés: Encountering Development. The making and unmaking of the Third World, Princeton, 1995.

Fukuyama, F., El fin de la historia y el último hombre, Barcelona, Planeta, 1992.

Goez, W., Translatio imperii, Tübingen, Mohr, 1958. 
Hardt, M., y Negri, A., Imperio, Barcelona, Paidós, 2005.

León Florido, F., "Translatio studiorum: traslado de los libros y diálogo de las civilizaciones en la Edad Media", en Revista general de información y documentación, vol. 15, no 2, 2005, 51-77.

Mann, M., El imperio incoherente. Estados Unidos y el nuevo orden internacional, Barcelona, Planeta, 2004;

Nájera, E., y Pérez Herranz, F. M. (eds.), La filosofía y la identidad europea, Valencia, Pre-textos, 2011.

Nebrija, A. de, Gramática sobre la lengua castellana (1492), ed. de C. Lozano, y Paginae nebrissenses, ed. de F. González Vega, Madrid y Barcelona, RAE y Galaxia Gutenberg, 2011.

Padua, M. de, Sobre el poder del imperio y del papa. El defensor menor. La transferencia del imperio, Madrid, Biblioteca Nueva, 2005.

Patińo Villa, C. A., Imperios contra Estados. La destrucción del orden internacional contemporáneo, Bogotá, Debate, 2017.

Sánchez Ferlosio, R., Esas Yndias equivocadas y malditas. Comentarios a la historia, Barcelona, Destino, 1994. Incluido en Ensayos y artículos, 2 vols., Barcelona, Destino, 1992, vol. II, pp. 517-803.

Scavino, D., "Sarmiento y la translatio imperii", en Estudios de Teoría Literaria, año 5, no 10, septiembre 2016, pp. 167-177.

Slaughter, S., y Leslie, L. L., Academic Capitalism: Politics, Policies, and the Entrepreneurial University, Baltimore, Johns Hopkins U P, 1997.

Slaughter, S., y Rhoades, G., Academic Capitalism and the New Economy; Markets, State, and Higher Education, Baltimore, Johns Hopkins U P, 2009.

Sousa Santos, B. de, La universidad en el siglo XXI. Para una reforma democrática y emancipadora de la universidad, Buenos Aires, Miño y Dávila, 2005.

Todd, E., Después del imperio. Ensayo sobre la descomposición del sistema norteamericano, Madrid, Akal, 2012.

Young, R. J. C., White Mitologies. Writing History and the West, Londres y Nueva York, Routledge, 1990, 2a ed. 2004.

DOI: http://doi.org/10.15366/bp2019.22.024

Bajo Palabra. II Época. No 22. Pgs: 443-458 
